Pacific Journal of Mathematics

ON THE EXTENSIONS OF A TORSION MODULE 


\section{ON THE EXTENSIONS OF A TORSION MODULE}

\section{R. J. NUNKE}

This paper concerns the structure of $\operatorname{Ext}(A, T)=\operatorname{Ext}_{R}^{1}(A, T)$ where $A$ is a torsion-free and $T$ is a torsion module over a Dedekind ring $R$. In the first section it is shown that for a given torsion-free module $A$ the structure of $\operatorname{Ext}(A, T)$ is completely determined by the basic subgroup of $T$. If in addition $T$ is primary the structure of $\operatorname{Ext}(A, T)$ depends on a single known invariant of $T$, called by Szele [4] the critical number. The rest of the paper is devoted to showing the nature of this dependence in the special case in which $A$ is the quotient field of $R$ and $T$ is primary. The problem reduces to that of computing the rank of certain complete modules over a discrete valuation ring. This computation is carried out in section two and the description of $\operatorname{Ext}(A, T)$ is given in section three.

Throughout the paper $R$ is assumed to be a Dedekind ring other than a field. A consequence of this assumption, used in section two, is that $R$ is infinite. An exact sequence $0 \rightarrow A^{\prime} \rightarrow A \rightarrow A^{\prime \prime} \rightarrow 0$ and a module $C$ give rise to two exact sequences. We follow $\mathrm{S}$. MacLane in calling the one beginning $0 \rightarrow \operatorname{Hom}\left(A^{\prime \prime}, C\right)$ the first exact sequence and the one beginning $0 \rightarrow \operatorname{Hom}\left(C, A^{\prime}\right)$ the second exact sequence.

1. In this section it is shown that whenever $A$ is torsion-free and $C$ is a torsion module, then the structure of $\operatorname{Ext}(A, C)$ depends only on the basic submodule of $C$.

Lemma 1.1. If $A, B, C$ are modules with $A$ torsion-free and if there is a homomorphism of $B$ into $C$ with divisible cokernel, then $\operatorname{Ext}(A, C)$ is a direct summand of $\operatorname{Ext}(A, B)$.

Proof. Suppose that $f: B \rightarrow C$ is a homomorphism with Coker $f=$ $C / \operatorname{Im} f$ divisible. Let $f$ be factored into an epimorphism $g$ followed by a monomorphism $h: f=h g$. We get two exact sequences

$$
\begin{aligned}
& 0 \longrightarrow \operatorname{Im} f \stackrel{h}{\longrightarrow} C \longrightarrow \text { Coker } f \longrightarrow 0 \\
& 0 \longrightarrow \operatorname{Ker} f \longrightarrow B \stackrel{g}{\longrightarrow} \operatorname{Im} \quad f \longrightarrow 0,
\end{aligned}
$$

and the relevant parts of the associated second exact sequences are 


$$
\begin{aligned}
& \operatorname{Hom}(A, \text { Coker } f) \longrightarrow h^{*} \\
& \operatorname{Ext}(A, \operatorname{Im} f) \stackrel{\operatorname{Ext}(A, C) \longrightarrow}{\longrightarrow} \operatorname{Ext}(A, \text { Coker } f) \longrightarrow 0 \\
& \operatorname{Ext}(A, \text { Koker } f) \longrightarrow \operatorname{Ext}(A, B) \stackrel{g^{*}}{\longrightarrow} \operatorname{Ext}(A, \operatorname{Im} f) \longrightarrow 0 .
\end{aligned}
$$

Since $A$ is torsion-free all the terms with Ext in them are divisible. But the divisibility of Coker $f$ implies that $\operatorname{Hom}(A$, Coker $f)$ is also divisible. For suppose that $\varphi: A \rightarrow$ Coker $f$ is a given homomorphism and $r$ is any nonzero element of $R$. Since $A$ is torsion-free, division by $r$ in $A$ is unique; hence there is a homomorphism $\psi: r A \rightarrow$ Coker $f$ defined by $\psi(r a)=\varphi(a)$ for $a$ in $A$. Since Coker $f$ is divisible $\psi$ can be extended to all of $A$. Then $r \psi(a)=\psi(r a)=\phi(a)$ so that $r \psi=\varphi$ and $\varphi$ is divisible by $r$.

Hence all the modules in the last two exact sequences are divisible and the images of the various homomorphisms are direct summands. In addition $\operatorname{Ext}(A, \operatorname{Coker} f)=0$ because Coker $f$ is divisible. It follows that $\operatorname{Ext}(A, C)$ is a direct summand of $\operatorname{Ext}(A, \operatorname{Im} f)$ which is in turn a direct summand of $\operatorname{Ext}(A, B)$.

Corollary 1.2. If $A$ is torsion-free and each of $B$ and $C$ has a homomorphism into the other with divisible cokernel, then

$$
\operatorname{Ext}(A, B) \approx \operatorname{Ext}(A, C) .
$$

Proof. A divisible $R$-module is a direct sum of submodules each of which is isomorphic to $Q$ or to a primary component of $Q / R$, the number of summands of each type being independent of the decomposition.

THEOREM 1.3. If $A$ is torsion-free, $C$ is a torsion module, and $B$ is a basic submodule of $C$, then

$$
\operatorname{Ext}(A, C) \approx \operatorname{Ext}(A, B) \text {. }
$$

Proof. A basic submodule of a torsion module is a pure submodule for which the factor module is divisible and which is a direct sum of cyclic modules. Hence there is a homomorphism of $B$ into $C$ with divisible cokernel. On the other hand Szele has shown in [4] that $B$ is a homomorphic image of $C$ (Szele's proof is for primary groups but the generalization to this case is trivial). Hence the hypotheses of Corollary 1.2 are satisfied and the conclusion follows.

Suppose now that $P$ is a prime ideal of $R$ and that $T$ is a $P$-primary module. The order ideal of an element $x$ of $T$ has the form $P^{e(x)}$ with $e(x)$ a nonnegative integer which we will call the exponential order of $x$. The submodule of $T$ consisting of those elements with exponential order $\leq 1$ is a vector space over the field $R / P$; its dimension will be 
called the $P$-rank of $T$ and will be denoted by $r_{P}(T)$. If $B$ is a basic submodule of $T$, the minimum of the numbers $r_{P}\left(P^{n} B\right)$ with $n$ ranging over the non-negative integers is independent of the choice of $B$ because the basic submodules of $T$ are all isomorphic. This number is thus an invariant of $T$. We shall follow Szele in calling it the critical number of $T$.

If the basic submodule $B$ of $T$ is decomposed into the direct sum of cyclic modules, then $r_{P}\left(P^{n} B\right)$ is the number of summands whose generators have exponential order $>n$. Hence $r_{P}\left(P^{n} B\right)$ finite implies that the orders of the elements of $B$ are bounded and the critical number of $T$ is then 0 . Thus the critical number of $T$ is either 0 or infinite, and if $0, B$ is a direct summand of $T$ which is therefore a direct sum of a divisible module and a module all of whose elements have bounded order.

THEOREM 1.4. Let $T$ be a P-primary module with critical number $\checkmark$ and let $A$ be torsion-free.

(i) If $\boldsymbol{\aleph}=0$, then $\operatorname{Ext}(A, T)=0$.

(ii) If $\aleph$ is infinite and $M$ is the direct sum of $\$$ copies of $\sum_{n} R^{\prime} P^{n}$, then $\operatorname{Ext}(A, T)$ and $\operatorname{Ext}(A, M)$ are isomorphic. Thus the module structure of $\operatorname{Ext}(A, T)$ depends only on the critical number of $T$.

Proof. Since the maximal divisible submodule of $T$ is a direct summand of $T$ and contributes neither to $\operatorname{Ext}(A, T)$ nor to the critical number of $T$, we may as well assume $T$ reduced. In the paragraph preceding the theorem it was shown that if $\boldsymbol{\psi}=0$, the orders of the elements of $T$ are bounded. Any extension of $T$ having a torsion-free factor module contains $T$ as a pure submodule. Hence it splits and $\operatorname{Ext}(A, T)=0$ in this case.

Suppose now that $\$$ is infinite and $M$ is the direct sum of $\$$ copies of $\sum_{n} R / P^{n}$. By Theorem $1.3 \operatorname{Ext}(A, T) \approx \operatorname{Ext}(A, B)$ where $B$ is a basic submodule of $T$. We write $B=\sum_{n} B_{n}$ where each $B_{n}$ is a direct sum of copies of $R / P^{n}$. There is a natural number $m$ such that $\boldsymbol{H}=r_{P}\left(P^{m} B\right)$ and $B=B^{\prime}+B^{\prime \prime}$ where $B^{\prime}$ is the sum of the $B_{n}$ with $n \leq m$ and $B^{\prime \prime}$ is the sum of the remaining $B_{n}$. Since $P^{m} B^{\prime}=0$ and $A$ is torsion-free, $\operatorname{Ext}\left(A, B^{\prime}\right)=0$. Then the additivity of Ext implies that $\operatorname{Ext}(A, B) \approx$ $\operatorname{Ext}\left(A, B^{\prime \prime}\right)$. The module $B^{\prime \prime}$ is the direct sum of cyclic modules and $r_{P}\left(B^{\prime \prime}\right)=r_{P}\left(P^{m} B^{\prime \prime}\right)=\boldsymbol{W}$ so that $B^{\prime \prime}$ is generated by $\boldsymbol{W}$ elements. Hence it is a homomorphic image of $M$. On the other hand $B^{\prime \prime}$ can be expressed as a direct sum $B^{\prime \prime}=C+\sum_{\gamma} C_{\gamma}$ where the summands $C_{\gamma}$ are in number and each $C_{\gamma}$ is the direct sum of a sequence of cyclic modules whose orders are strictly increasing. It follows that $M$ is also a homomorphic image of $B^{\prime \prime}$, hence $\operatorname{Ext}\left(A, B^{\prime \prime}\right) \approx \operatorname{Ext}(A, M)$ by Corollary 1.2. This proves (ii). 
2. In this section we assume that $R$ is a discrete valuation ring with prime $p$. If $M$ is an $R$-module for which the submodules $p^{n} M$ have intersection 0 (i. e. if $M$ has no elements of infinite height), then these submodules are a base at 0 for a topology called the p-adic topology. The completion of $M$ in this topology will be denoted by $M^{*}$. The $p$ adic topology on $M$ induces a topology on each submodule $N$ which may or may not coincide with the $p$-adic topology on $N$. The two topologies will certainly coincide if $N$ is pure in $M$ for then $p^{n} N=N \cap p^{n} M$ for all $n$.

The problem to be solved in this section is that of determining the rank of $M^{*}$ where $M$ is a direct sum of copies of $\Sigma_{n} R / p^{n} R$.

$A$ subset $X$ of an $R$-module $A$ is called independent if $r_{1} x_{1}+\cdots+r_{n} x_{n}=$ 0 implies $r_{1}=\cdots=r_{n}=0$ whenever $x_{1}, \cdots, x_{n}$ are distinct elements of $X$ and $r_{1}, \cdots, r_{n}$ are elements of $R$. The cardinal $|X|$ of a maximal independent subset of $A$ is an invariant of $A$ called its rank (denoted by $r(A)$ ); the rank of $A$ is in fact the dimension of $A \otimes_{R} Q$ as a vector space over $Q$. The rank formula

$$
r(A)=r(B)+r(A / B)
$$

holds for any $R$-modules $A$ and $B$ with $B$ a submodule of $A$. If $A$ is torsion-free its cardinal $|A|$ and its rank are connected by the relation

$$
|A|=r(A)|R| \text {. }
$$

In particular $r(A)=|A|$ wherenever $A$ is torsion-free and $r(A) \leq|R|$. (The properties mentioned is this paragraph hold for any Dedekind ring.)

LEMMA 2.1. If $M=\Sigma_{\gamma} M_{\gamma}$ is the direct sum of the modules $M_{\gamma}$, each of which is without elements of infinite height then $M^{*}$ is isomorphic to the submodule of the direct product $\Pi_{\gamma} M_{\gamma}^{*}$ consisting of those sequences $u=\left(u_{\gamma}\right)$ such that $\left(^{*}\right)$ for each natural number $n, u_{\gamma} \in p^{n} M_{\gamma}^{*}$ for all but a finite set of indices.

The condition $\left(^{*}\right)$ implies that $u_{\gamma}=0$ for all but a countable set of indices.

Proof. For each index $\gamma M_{\gamma}$ is pure in $M$ which is pure in $M^{*}$. Hence $M_{\gamma}$ is pure in $M^{*}$. By Lemma 20 of [2] the closure $M_{\bar{\gamma}}$ of $M_{\gamma}$ in the $p$-adic topology is also pure in $M^{*}$. Therefore $M^{*}$ induces the $p$-adic topology on $M_{\bar{\gamma}}$ and, since a closed subspace of a complete space is complete, $M_{\bar{\gamma}}=M_{\gamma}^{*}$.

We next show that the sum $\Sigma_{\gamma} M_{\gamma}^{*} \subseteq M^{*}$ is direct. Suppose $\Sigma_{\gamma} x_{\gamma}=0$ where $x_{\gamma} \in M^{*}$ and $\gamma$ belongs to a finite set $\sigma$ of indices. For each natural number $n$ and each $\gamma \in \sigma$ there is an $x_{\gamma_{n}} \in M_{\gamma}$ such that $x_{\gamma_{n}}-x_{\gamma} \in p^{n} M_{\gamma}^{*}$, hence $\Sigma_{\gamma} x_{\gamma n}=\Sigma_{\gamma}\left(x_{\gamma_{n}}-x_{\gamma}\right) \in p^{n} M^{*}$. Since $\Sigma_{\gamma} M_{\gamma}$ is pure in $M^{*}$ it is pure 
in $\Sigma_{\gamma} M_{\lambda}^{*}$ so that $\Sigma_{\gamma} x_{\gamma n} \in\left(\Sigma_{\gamma} M_{\gamma}\right) \cap p^{n} \Sigma_{\gamma} M_{\gamma}^{*}=p^{n} \Sigma_{\gamma} M_{\gamma}$. Then $x_{\gamma n} \in p^{n} M$ for each $\gamma \in \sigma$ because the sum $\Sigma_{\gamma} M_{\gamma}$ is direct. Thus for each $\gamma \in \sigma, x_{\gamma_{n}} \rightarrow 0$ and $x_{\gamma}=0$.

Let $S$ be the submodule of $\Pi_{\gamma} M_{\gamma}^{*}$ defined by $\left({ }^{*}\right)$. We shall define an isomorphism $\varphi$ of $M^{*}$ onto $S$. Let $x$ be any element of $M^{*}$. Since $\Sigma_{\gamma} M_{\gamma}^{*}$ is dense in $M^{*}$ there is, for each natural number $n$, an element $x_{n} \in \Sigma_{\gamma} M_{\gamma}^{*}$ such that $x_{n}-x \in p^{n} M^{*}$. We express each $x_{n}$ as a sum $x_{n}=$ $\Sigma_{\gamma} x_{\gamma_{n}}$ with $x_{\gamma_{n}} \in M_{\gamma}^{*}$ where $x_{\gamma n}=0$ for all $\gamma$ not in some finite set $\tau_{n}$. Since $x_{n}$ converges to $x$, the arguments of the preceding paragraph show that, for each $\gamma, x_{\gamma n}$ converges to some $u_{\gamma} \in M^{*}$. It is easily shown that the elements $u_{\gamma}$ depend only on $x$. We set $\varphi(x)=\left(u_{\gamma}\right)$.

It is necessary to show that $u$ lies in $S$. Consider a fixed natural number $i$ and assume that $\gamma$ is not in $\tau_{i}$ so that $x_{\gamma_{i}}=0$. Then, for $j>i$, $x_{\gamma_{j}}=x_{\gamma j}-x_{\gamma_{i}} \in p^{i} M^{*} \cap M_{\gamma}^{*}=p^{i} M_{\gamma}^{*}$. Passing to the limit we have $u_{\gamma} \in p^{i} M_{\gamma}^{*}$ because $p^{i} M_{\gamma}^{*}$ is closed in $M^{*}$. Since each $\tau_{i}$ is finite, $u_{\gamma}$ satisfies $\left(^{*}\right)$ and is in $S$ as required.

To prove $\phi$ epimorphic suppose $u \in S$. For each $n$ let $\tau_{n}$ be a finite set of indices such that $u_{\gamma} \in p^{n} M_{\gamma}^{*}$ for all $\gamma$ not in $\tau_{n}$ and let $x_{n}$ be the sum (in $M^{*}$ ) of the $u_{\gamma}$ for $\gamma \in \tau_{n}$. The existence of $\tau_{n}$ is insured by $\left(^{*}\right.$ ). Since $\tau_{n} \subseteq \tau_{m}$ for $m \leq n, x_{m}-x_{n} \in p^{n} M^{*}$. Hence the $x_{n}$ converge to an element $x$ in $M^{*}$. Moreover $x_{n}-x \in p^{n} M^{*}$. An examination of the definition of $\varphi$ shows that $x_{\gamma n}=u_{\gamma}$ if $\gamma \in \tau_{n}$ and $x_{\gamma n}=0$ otherwise. Hence $\varphi(x)=u$ and $\varphi$ is epimorphic.

Finally suppose that $\varphi(x)=0$. Refering to the definition of $\rho$ we have, for fixed $n$ and all $i>n,\left(\Sigma_{\gamma i}-x_{\gamma n}\right)=x_{i}-x_{n} \in p^{n} M^{*}$. Since $\Sigma_{\gamma} M_{\gamma}^{*}$ is pure in $M^{*}$ and the sum is direct, this implies that $x_{\gamma_{i}}-x_{\gamma_{n}} \in p^{n} M_{\gamma}^{*}$ for each index $\gamma$ and each $i>n$. We are assuming all $u_{\gamma}=0$ so that $x_{\gamma_{i}} \in p^{n} M_{\gamma}^{*}$ for large $i$, hence $x_{\gamma n} \in p^{n} M_{\gamma}^{*}$. But then $x_{n}=\Sigma_{\gamma} x_{\gamma n} \in p^{n} M^{*}$ and $x_{n} \rightarrow 0, x=0$. This shows that $\phi$ is a monomorphism and completes the proof.

Lemma 2.3. If $M=\Pi_{\gamma} M_{\gamma}$ where $\gamma$ ranges over a set of cardinal $\aleph$ and the $M_{\gamma}$ are all torsion-free with the same rank, then

$$
r(M)=\left|M_{\gamma}\right|^{\aleph} .
$$

Proof. Note first that for each $\gamma\left|M_{\gamma}\right|=\left.r\left(M_{\gamma}\right)\right|^{\vee} R \mid$ so that all the $M_{\gamma}$ have the same power. If we can show that $r(M) \geq|R|$, then $r(M)=$ $|M|=\left|M_{\gamma}\right|^{\wedge}$ as required.

Suppose the indices are the natural numbers and that each $M_{\gamma}=R$. Consideration of a suitable Vandermonde determinant shows that the elements $\left(1, r, r^{2}, \cdots\right) \in M$ with $r$ ranging over $R$ are independent so that $r(M) \geq|R|$ in this case. In the general case $\boldsymbol{W}$ is infinite and each $M_{\gamma}$ contains a copy of $R$ so that $M$ contains a countable product of copies of $R$, hence $r(M) \geq|R|$ in all cases. 
Lemma 2.3. Suppose that $N$ is a submodule of $M$ and that, for each natural number $n, M_{n}$ and $N_{n}$ are copies of $M$ and $N$ respectively. If $\varphi: \Pi_{n} M_{n} \rightarrow M$ is a homomorphism such that $\varphi^{-1}(N) \subseteq \Pi_{n} N_{n}$, then

$$
r(M / N)=r(M / N)^{N_{0}} .
$$

Proof. Since $\phi$ maps $\phi^{-1}(N)$ into $N$, it induces a monomorphism

$$
0 \rightarrow \Pi_{n} M_{n} / \varphi^{-1}(N) \rightarrow M / N .
$$

Since $\varphi^{-1}(N) \subseteq \Pi_{n} N_{n}$, there is an epimorphism

$$
I_{n} M_{n} / \mathcal{P}^{-1}(N) \rightarrow \Pi_{n}\left(M_{n} / N_{n}\right) \rightarrow 0 .
$$

Rank does not increase on passing to submodules or to homomorphic images, hence (1) and (2) imply

$$
r(M / N) \geq r\left(\Pi_{n} M_{n} / \varphi^{-1}(N)\right) \geq r\left(\Pi_{n}\left(M_{n} / N_{n}\right)\right) .
$$

By the definition of rank $M / N$ contains a free module $F$ such that $r(F)=r(M / N)$. For each $n$ let $F_{n}$ be a copy of $F$ in $M_{n} / N_{n}$. Then $I_{n} F_{n} \subseteq \Pi_{n}\left(M_{n} / N_{n}\right)$ and Lemma 2.2 implies

$$
r\left(\Pi_{n}\left(M_{n} \mid N_{n}\right)\right) \geq r\left(\Pi_{n} F_{n}\right)=|F|^{\aleph_{0}} \geq r(F)^{\aleph_{0}}=r(M / N)^{\aleph_{0}} .
$$

Thus (3) and (4) imply the conclusion of the lemma.

Theorem 2.4. If $M$ is the direct sum of $\boldsymbol{~ c o p i e s ~ o f ~} \Sigma_{n} R / p^{n} R$, then $r\left(M^{*}\right)=(\boldsymbol{\aleph}|R|)^{\wedge}$.

Proof. We first consider the case $\boldsymbol{\aleph}=1$. It will be convenient to replace $R / p^{n} R$ by the isomorphic module $R\left(p^{n}\right)$ which consists of all elements of $Q / R$ annihilated by $p^{n}$, for then $R\left(p^{n}\right) \subseteq R\left(p^{m}\right)$ for all $m \geq n$. Each element $a \neq 0$ in $R\left(p^{n}\right)$ has a height $h_{n}(a)$ in $R\left(p^{n}\right)$ where $h_{n}(a)=i$ if $a \in p^{i} R\left(p^{n}\right)$ but a is not in $p^{i+1} R\left(p^{n}\right)$. The height and exponential order of $a$ are related by $h_{n}(a)+e(a)=n$. We let $C=\Sigma_{n} R\left(p^{n}\right)$ and $D=$ $\Pi_{n} R\left(p^{n}\right)$. Then $C^{*}$ consists of those elements $x=\left(x_{n}\right) \in D$ such that $h_{n}\left(x_{n}\right)$ goes to $\infty$ with $n$.

We show first that $r\left(C^{*}\right)=r(D)$. The inequality $r\left(C^{*}\right) \leq r(D)$ holds because $C^{*} \subseteq D$. To prove the opposite inequality we define $\rho: D \rightarrow C^{*}$ by

$$
\rho(x)_{n}=\left\{\begin{array}{lll}
0 & \text { if } & n=2 k+1 \\
x_{k} & \text { if } & n=2 k .
\end{array}\right.
$$

Since $R\left(p^{k}\right) \subseteq R\left(p^{2 k}\right), \rho$ is a homomorphism into $D$. Since $e\left(x_{k}\right) \leq k$ and $h_{2 k}\left(x_{k}\right)+e\left(x_{k}\right)=2 k, h_{2 k}\left(x_{k}\right) \geq k$ so that $\rho(x)$ lies in $C^{*}$. The map $\rho$ is clearly a monomorphism so $r(D) \leq r\left(C^{*}\right)$ as required. 
The next step is to show that

$$
r(D)=r(D)^{\aleph_{0}} \text {. }
$$

Let $\sigma_{1}, \sigma_{2}, \cdots$ be an infinite partition of the set of natural numbers into infinite subsets. For each $n$ let $D_{n}$ be a copy of $D$. An element $u \in I_{n} D_{n}$ is a sequence $\left(u_{1}, u_{2}, \cdots\right)$ with $u_{n}=\left(u_{n i}\right) \in D$. We define $\xi: \Pi_{n} D_{n} \rightarrow D$ by $\xi(u)_{k}=u_{n i}$ if $k$ is the $i$ th element of $\sigma_{n} ; u_{n i} \in R\left(p^{k}\right)$ because $k \geq i$. The hypotheses of Lemma 2.3 are satisfied with $M=D$ and $N=0$ which shows that $r(D)=r(D)^{\times 0}$.

The module $D$ can be represented as the module of all infinite sequences $\left(x_{1}, x_{2}, \cdots\right)$ of elements of $R$ modulo the sequences of the form $\left(b_{1} p, b_{2} p^{2}, b_{3} p^{3}, \cdots\right)$. Thus Lemma 2.2 and the fact that rank does not increase on passing to homomorphic images imply that $r(D) \leq|R|^{*_{0}}$. We shall show that $r(D) \geq|R|$. Then $r(D)=r(D)^{\aleph_{0}} \geq|R|^{\aleph_{0}}$ and we get

$$
r(D)=|R| \aleph_{0} .
$$

To show that $r(D) \geq|R|$ let $\alpha(r)=\left(1, r, r^{2}, \cdots\right)$ for each $r \in R$ and let $\bar{\alpha}(r)$ be the image of $\alpha(r)$ in $D$. We show that the elements $\bar{\alpha}(r)$ for $r \in R-(p)$ are independent. Suppose $r_{1}, \cdots, r_{n}$ are distinct elements of $R$ not in $(p)$, and suppose $a_{1}, \cdots, a_{n} \in R$ such that

$$
a_{1} \bar{\alpha}\left(r_{1}\right)+\cdots+a_{n} \bar{\alpha}\left(r_{n}\right)=0 .
$$

Then elements $b_{1}, b_{2}, \cdots$ exist in $R$ such that

$$
a_{1} \alpha\left(r_{1}\right)+\cdots+a_{n} \alpha\left(r_{n}\right)=\left(b_{1} p, b_{2} p^{2}, \cdots\right) .
$$

Hence, for each $k$, the $a_{i}$ satisfy a system of $n$ equations

$$
\begin{aligned}
& a_{1} r_{1}^{k}+\cdots+a_{n} r_{n}^{k}=b_{k} p^{k} \\
& \text { } \ldots \ldots \ldots \ldots \ldots \ldots \ldots \ldots \ldots \ldots \\
& a_{1} r_{1}^{k+n-1}+\cdots+a_{n} r_{n}^{k+n-1}=b_{k+n-1} p^{k+n-1} .
\end{aligned}
$$

The determinant $\Delta$ of this system is $r_{1}^{k} \cdots r_{n}^{k} d$ where $d$ is the Vandermonde determinant of $r_{1}, \cdots, r_{n} ; d \neq 0$ because the $r$ 's are distinct. We set $d=p^{m} s$ with $s$ prime to $p$ and $t=r_{1}^{k} \cdots r_{n}^{k} s$. Then $\Delta=p^{m} t$ where $t$ is prime to $p$ because $r_{1}, \cdots, r_{n}, s \in R-(p)$. Then by Cramer's rule each $a_{i}$ satisfies an equation of the form $p^{m} t a_{i}=p^{k} c_{i}$. Hence, for $k>m$, $p^{k-m}$ divides $t a_{i}$ and therefore divides $a_{i}$ because it is prime to $t$. Since this is true for all $k>m, a_{i}=0$ for each $i$. Therefore the $\bar{\alpha}(r)$ with $r$ ranging over $R-(p)$ is an independent subset of $D$ so $r(D) \geq|R-(p)|$. But $R-(p)$ is the disjoint union of cosets of $(p)$ so that $\mid R-(p) \geq$ $|(p)|=|R|$; hence $|R-(p)|=|R|$. 
We now have $r\left(C^{*}\right)=r(D)=|R|^{N_{0}}$ which completes the proof in the case $\boldsymbol{K}=1$.

Now suppose $\boldsymbol{\aleph}$ arbitrary, let $\Gamma$ be a set with cardinal $\boldsymbol{\aleph}$ and let $M=\Sigma_{\gamma} M_{\gamma}$ where, for each $\gamma \in \Gamma, M_{\gamma}=C=\Sigma_{n} R\left(p^{n}\right)$. In view of Lemma 2.1 and the remark following it $M^{*}$ is contained in the submodule $A$ of all sequences $x \in \Pi_{\gamma} M_{\gamma}^{*}$ with $x_{\gamma}=0$ for all but a countable number of indices. Each such sequence is determined by the set $\sigma$ of indices $\gamma$ such that $x_{\gamma} \neq 0$ and a function $f: \sigma \rightarrow C^{*}-\{0\}$. From this it follows easily that $|A| \leq\left(\boldsymbol{\aleph}\left|C^{*}\right|\right)^{\boldsymbol{N}_{0}}$. Since $C^{*} \subseteq D$ and $D$ is a homomorphic image of the direct product of $\boldsymbol{\aleph}_{0}$ copies of $R,\left|C^{*}\right| \leq|R|^{\boldsymbol{\aleph}_{0}}$. Since $|R|^{\aleph_{0}}=r\left(C^{*}\right) \leq\left|C^{*}\right|$ we have $\left|C^{*}\right|=|R|^{\aleph_{0}}$. Hence

$$
r\left(M^{*}\right) \leq r(A) \leq|A| \leq(\boldsymbol{\aleph}|R|)^{\aleph_{0}} .
$$

Using Lemma 2.1 again we have $\Sigma_{\gamma} M_{\gamma}^{*} \subseteq M^{*}$ so that

$$
r\left(M^{*}\right) \geq r\left(\Sigma_{\gamma} M_{\gamma}^{*}\right)=|\Gamma| r\left(C^{*}\right)=\boldsymbol{\aleph}|R|^{\boldsymbol{N}_{0}} .
$$

These last two sets of inequalities combine to give

$$
\boldsymbol{\aleph}|R|^{\boldsymbol{\aleph}_{0}} \leq r\left(M^{*}\right) \leq(\boldsymbol{\aleph}|R|)^{\boldsymbol{\aleph}_{0}} .
$$

If $\boldsymbol{X}$ is finite this completes the proof. If $\boldsymbol{X}$ is infinite, the proof will be complete once we show that $r\left(M^{*}\right)^{*_{0}}=r\left(M^{*}\right)$. To show this assume $\aleph$ infinite and partition the index set $\Gamma$ into a countable sequence $\Gamma_{1}$, $\Gamma_{2}, \cdots$ of disjoint subsets such that $\left|\Gamma_{n}\right|=|\Gamma|=\boldsymbol{\aleph}$ and set $M_{n}=$ $\Sigma\left\{M_{\gamma} \mid \gamma \in \Gamma_{n}\right\}$. Then $M_{n} \approx M$ and $M_{n}^{*} \approx M^{*}$ for each $n$. Our purpose will be achieved if we can define a monomorphism $\varphi: \Pi_{n} M_{n}^{*} \rightarrow M^{*}$, for then $\varphi^{-1}\left(t M^{*}\right)=t\left(\Pi_{n} M_{n}^{*}\right) \subseteq \Pi_{n} t M_{n}^{*}$, where $t M^{*}$ is the torsion submodule of $M^{*}$. Now Lemma 2.3 applies to give $r\left(M^{*} / t M^{*}\right)=r\left(M^{*} / t M^{*}\right)^{\aleph_{0}}$. But $r\left(M^{*}\right)=r\left(M^{*} / t M^{*}\right)$ so $r\left(M^{*}\right)=r\left(M^{*}\right)^{\boldsymbol{N}_{0}}$.

Earlier in the proof of this theorem we defined a monomorphism $\rho: D \rightarrow C^{*}$. For each $k$ we now define a monomorphism $\psi_{k}: D \rightarrow D$ by

$$
\psi_{k}(x)_{i}=\left\{\begin{array}{l}
0, i \leq k \\
x_{i-k}, i>k
\end{array}\right.
$$

For $i>k$ we have $e\left(x_{i-n}\right) \leq i-k$ so that $h_{i}\left(x_{i-k}\right)=i-e\left(x_{i-k}\right) \geq k$. Hence $\psi_{k}(D) \subseteq p^{k} D$ so that $\rho \psi_{k}$ maps $D$ into $p^{k} C^{*}$. We define $\varphi_{k}: C^{*} \rightarrow p^{k} C^{*}$ to be the restriction of $\rho \psi_{k}$ to $C^{*}$ and note that it is a monomorpoism.

We now use Lemma 2.1 to identify $M^{*}$ with the submodule of $\Pi_{\gamma} M_{\gamma}^{*}$ described by the condition $\left(^{*}\right)$. An element $x$ of $I_{n} M_{n}^{*}$ is a sequence $\left(x_{1}, x_{2}, \cdots\right)$ where $x_{n} \in M_{n}^{*} \subseteq \Pi\left\{M_{\gamma}^{*} \mid \gamma \in \Gamma_{n}\right\}$. We define $\phi$ by $\phi(x)_{\gamma}=\varphi_{n}\left(x_{n \gamma}\right)$ for $\gamma \in \Gamma_{n}$. Then $\varphi: I_{n} M_{n}^{*} \rightarrow \Pi_{\gamma} M_{\gamma}^{*}$ and is a monomorphism because each $\mathcal{P}_{n}$ is one. There remains the task of showing that $\varphi(x)$ lies in $M^{*}$. Let $n$ be a natural number. For each $k<n$ there is by Lemma 2.1 a finite subset $\tau_{k}$ of $\Gamma_{k}$ such that $x_{k \gamma} \in p^{n} M_{\gamma}^{*}$ for $\gamma \in \Gamma_{k}$ 
but not in $\tau_{k}$. By the definition of $\varphi_{k}, \varphi_{k}\left(x_{k \gamma}\right) \in p^{n} M_{\gamma}^{*}$ for all $\gamma \in \Gamma_{k}$ with $k \geq n$. Hence $\varphi(x)_{\gamma} \in p^{n} M_{\gamma}^{*}$ for all not in $\tau_{1} \cup \cdots \cup \tau_{n-1}$ which is a finite set. Thus $\varphi(x)$ satisfies $\left(^{*}\right)$ of Lemma 2.1 and is in $M^{*}$ as required.

3. Let $R$ once more be an arbitrary Dedekind ring and let $P$ be a prime ideal of $R$. For any $R$-module $T, \operatorname{Ext}(Q, T)$ is a vector space over $Q$ and is therefore completely described by its dimension over $Q$ or equivalently its rank over $R$. According to Theorem 1.4 this dimension is a function of the critical number of $T$ if $T$ is primary.

THEOREM 3.1. If $T$ is a P-primary $R$-module with infinite critical number $\boldsymbol{\aleph}$, then the rank of $\operatorname{Ext}(Q, T)$ is $(\boldsymbol{K}|R|)^{\aleph_{0}}$.

Proof. In order to make the results of section two available we change rings. The module $T$, being $P$-primary, can be considered as a module over the ring $S$ consisting of all elements of the form $a / b$ in $Q$ with $a$ and $b$ in $R$ and $b$ prime to $P$. The theory of $P$-primary modules is left unchanged by the shift from $R$ to $S$. In particular the critical number of $T$ is $\boldsymbol{K}$ in both cases.

Since $S$ is torsion-free as an $R$-module Proposition 4.1.3. of [1] applies to give a natural isomorphism

$$
\operatorname{Ext}_{R}(Q, T) \approx \operatorname{Ext}_{S}\left(S \otimes_{R} Q, T\right) .
$$

Since $R$ and $S$ have the same quotient field $Q, Q=S \otimes_{R} Q$ and

$$
\operatorname{Ext}_{R}(Q, T) \approx \operatorname{Ext}_{S}(Q, T) .
$$

These are both vector spaces over $Q$ and the isomorphism is a $Q$-isomorphism; hence the two modules have the same dimension over $Q$. Let $M$ be the direct sum of $\gg$ copies of $\Sigma_{n} S / p^{n} S$ where $p$ is the prime of $S$. According to Theorem 1.4

$$
\operatorname{Ext}_{S}(Q, T) \approx \operatorname{Ext}_{S}(Q, M) .
$$

Since $M$ is a basic submodule of $t M^{*}$, Theorem 1.3 gives

$$
\operatorname{Ext}_{S}(Q, M) \approx \operatorname{Ext}_{S}\left(Q, t M^{*}\right) .
$$

By Theorem 7.4 of [3], $\operatorname{Ext}_{S}\left(Q, M^{*}\right)=0$ because $M^{*}$ is complete, while $\operatorname{Hom}_{S}\left(Q, M^{*}\right)=0$ because $M^{*}$ is reduced. Hence the second exact sequence associated with $Q$ and $0 \rightarrow t M^{*} \rightarrow M^{*} \rightarrow M^{*} / t M^{*} \rightarrow 0$ reduces to

$$
0 \rightarrow \operatorname{Hom}_{S}\left(Q, M^{*} / t M^{*}\right) \rightarrow \operatorname{Ext}_{s}\left(Q, t M^{*}\right) \rightarrow 0 .
$$

Since $M^{*} / t M^{*}$ is torsion-free divisible

$$
\operatorname{Hom}_{S}\left(Q, M^{*} / t M^{*}\right) \approx M^{*} / t M^{*} .
$$


It follows that $\operatorname{Ext}_{R}(Q, T)$ and $M^{*} / t M^{*}$ have the same dimension over $Q$. This dimension is $(\boldsymbol{S}|S|)^{N_{0}}$ by Theorem 2.5. Moreover $|R|=|S|$. Hence the theorem is proved.

Since the integers are the most important example of a Dedekind ring it is appropriate to interpret the last theorem for this special case. Since rank and cardinality coincide for torsion-free abelian groups of infinite rank, we can say that if $T$ is a p-primary abelian group with infinite critical number $\boldsymbol{\aleph}$, there are $\boldsymbol{\aleph}^{*}$ inequivalent extensions of $T$ by the rational numbers.

\section{REFERENCES}

1. H. Cartan and S. Eilenberg, Homological Algebra, Princeton 1956.

2. I Kaplansky, Infinite Abelian Groups, Ann Arbor, 1954.

3. R. J. Nunke, Modules of extensions over Dedekind rings. Ill. Journ. of Math., 3, (1959), 222-241.

4. T. Szele, On the basic subgroups of abelian p-groups, Acta. Math. Acad. Sci. Hung., 5 (1954), 129-141.

UNIVERSITY OF WASHINGTON 


\section{PACIFIC JOURNAL OF MATHEMATICS}

\section{EDITORS}

David Gilbarg

Stanford University

Stanford, California

F. H. Brownell

University of Washington

Seattle 5, Washington
A. L. Whiteman

University of Southern Californıa Los Angeles 7, California

L. J. PaIge

University of California

Los Angeles 24, California

\section{ASSOCIATE EDITORS}
E. F. BECKENBACH
T. M. CHERRY
D. DERRY

E. HEWITT
A. HORN
L. NACHBIN
M. OHTSUKA

H. L. ROYDEN

M. M. SCHIFFER
E. SPANIER

E. G. STRAUS

F. WOLF

\section{SUPPORTING INSTITUTIONS}

\author{
UNIVERSITY OF BRITISH COLUMBIA \\ CALIFORNIA INSTITUTE OF TECHNOLOGY \\ UNIVERSITY OF CALIFORNIA \\ MONTANA STATE UNIVERSITY \\ UNIVERSITY OF NEVADA \\ NEW MEXICO STATE UNIVERSITY \\ OREGON STATE COLLEGE \\ UNIVERSITY OF OREGON \\ OSAKA UNIVERSITY \\ UNIVERSITY OF SOUTHERN CALIFORNIA
}

\author{
STANFORD UNIVERSITY \\ UNIVERSITY OF TOKYO \\ UNIVERSITY OF UTAH \\ WASHINGTON STATE COLLEGE \\ UNIVERSITY OF WASHINGTON \\ AMERICAN MATHEMATICAL SOCIETY \\ CALIFORNIA RESEARCH CORPORATION \\ HUGHES AIRCRAFT COMPANY \\ SPACE TECHNOLOGY LABORATORIES \\ NAVAL ORDNANCE TEST STATION
}

Mathematical papers intended for publication in the Pacific Journal of Mathematics should be typewritten (double spaced), and the author should keep a complete copy. Manuscripts may be sent to any one of the four editors. All other communications to the editors should be addressed to the managing editor, L. J. Paige at the University of California, Los Angeles 24, California.

50 reprints per author of each article are furnished free of charge; additional copies may be obtained at cost in multiples of 50 .

The Pacific Journal of Mathematics is published quarterly, in March, June, September, and December. The price per volume (4 numbers) is $\$ 12.00$; single issues, $\$ 3.50$. Back numbers are available. Special price to individual faculty members of supporting institutions and to individual members of the American Mathematical Society: $\$ 4.00$ per volume; single issues, $\$ 1.25$.

Subscriptions, orders for back numbers, and changes of address should be sent to Pacific Journal of Mathematics, 2120 Oxford Street, Berkeley 4, California.

Printed at Kokusai Bunken Insatsusha (International Academic Printing Co., Ltd.), No. 6, 2-chome, Fujimi-cho, Chiyoda-ku, Tokyo, Japan.

PUBLISHED BY PACIFIC JOURNAL OF MATHEMATICS, A NON-PROFIT CORPORATION

The Supporting Institutions listed above contribute to the cost of publication of this Journal, but they are not owners or publishers and have no responsibility for its content or policies. 


\section{Pacific Journal of Mathematics}

\section{Vol. 10, No. $2 \quad$ October, 1960}

Maynard G. Arsove, The Paley-Wiener theorem in metric linear spaces ........

Robert (Yisrael) John Aumann, Acceptable points in games of perfect

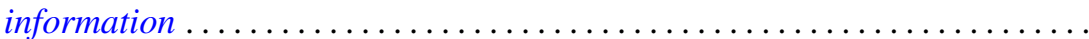

A. V. Balakrishnan, Fractional powers of closed operators and the semigroups generated by them ... . . . . . . . . . . . . . . . . . . . . . . . . . . . . 419

Dallas O. Banks, Bounds for the eigenvalues of some vibrating systems . . . . . 439

Billy Joe Boyer, On the summability of derived Fourier series . . . . . . . . . . . 475

Robert Breusch, An elementary proof of the prime number theorem with

remainder term ...................................

Edward David Callender, Jr., Hölder continuity of $n$-dimensional

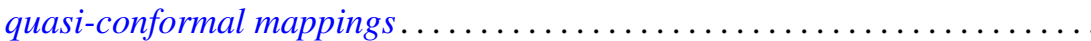

L. Carlitz, Note on Alder's polynomials ......................... 517

P. H. Doyle, III, Unions of cell pairs in $E^{3} \ldots \ldots \ldots \ldots \ldots \ldots \ldots \ldots \ldots \ldots \ldots \ldots . \ldots 21$

James Eells, Jr., A class of smooth bundles over a manifold . . . . . . . . . . . . 525

Shaul Foguel, Computations of the multiplicity function . . . . . . . . . . . . . . 539

James G. Glimm and Richard Vincent Kadison, Unitary operators in

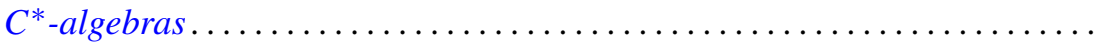

Hugh Gordon, Measure defined by abstract $L_{p}$ spaces . . . . . . . . . . . 557

Robert Clarke James, Separable conjugate spaces ....................

William Elliott Jenner, On non-associative algebras associated with bilinear forms

Harold H. Johnson, Terminating prolongation procedures

John W. Milnor and Edwin Spanier, Two remarks on fiber homotopy type .

Donald Alan Norton, A note on associativity . .

Ronald John Nunke, On the extensions of a torsion module.

Joseph J. Rotman, Mixed modules over valuations rings . . . . .

A. Sade, Théorie des systèmes demosiens de groupoï des . .

Wolfgang M. Schmidt, On normal numbers . .

661

Berthold Schweizer, Abe Sklar and Edward Oakley Thorp, The metrization of

statistical metric spaces

John P. Shanahan, On uniqueness questions for hyperbolic differential

equations

A. H. Stone, Sequences of coverings

Edward Oakley Thorp, Projections onto the subspace of compact operators

L. Bruce Treybig, Concerning certain locally peripherally separable spaces

Milo Wesley Weaver, On the commutativity of a correspondence and a

permutation

David Van Vranken Wend, On the zeros of solutions of some linear complex

differential equations. 